\title{
Breast Filariasis
}

\section{A MASHANKAR, K KHOPKAR, A PARIHAR, P SALKADE}

Ind J Radiol Imag 2005 15:2:203-204

Keywords: Filariasis, Breast, Mammography, USG

\section{INTRODUCTION}

Filarial affection of the breast is an uncommon condition. It usually presents with a mass in breast, which shows calcification on mammograms.

Wuchereria bancrofti and Brugia malayi are the prime causative micro-organisms in the etiology of the disease.

Lymphatic filariasis is the commonest involvement in the tropics leading to lymphaedema, hydrocele and elephantiasis.

Tropical pulmonary eosinophilia is a less common manifestation.

\section{CASE REPORT}

25yrs old woman patient presented with a painful lump of three months duration in the right breast.

The lump showed gradual increase in size over this period of three months.

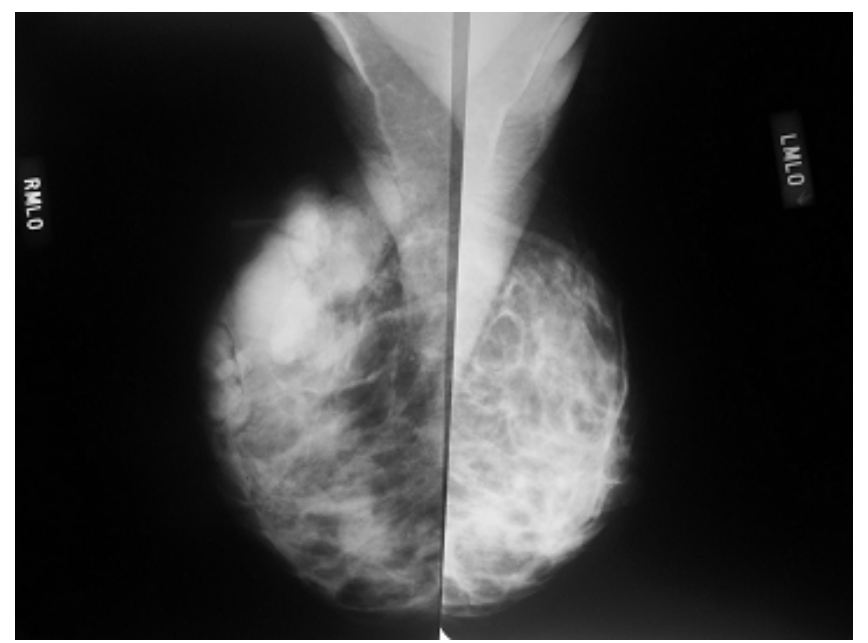

Fig 1

After initial clinical examination patient was subjected to film screen mammography. Routine blood examination reports revealed evidence of eosinophilia. Mammography revealed a lobulated mass lesion in the upper outer quadrant of right breast (Fig 1). No calcification was observed.

Sonomammography revealed a cluster of multiple, thin walled, fluid filled cystic lesions in the region of the palpable mass.

The largest cyst showed a cluster of elongated worm like echogenic structures displaying slow, repetitive dancing movements (Fig 2). The entire ball of these structures also showed free movements within the cyst. No obvious calcification was seen in the mass.

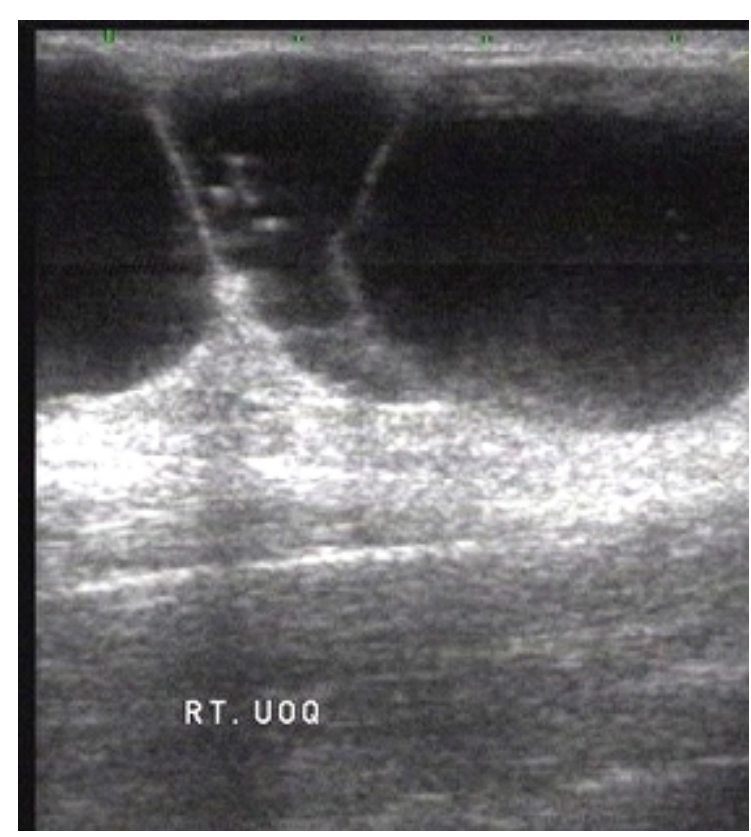

Fig 2

From the Mandakini Imaging Centre, 90 feet Road, Ghatkopar, Mumbai-77

Request for Reprints: Dr. A.S. Mashankar, Consultant Radiologist, Mandakini Imaging Centre, 90 feet Road, Opp Garodia Palace, Ghatkopar (E), Mumbai - 400077

Received 10 September 2004; Accepted 20 February 2005 
A provisional diagnosis of filariasis of right breast was made, based on the characteristic dancing movement of the micro-organism within the cyst.

The patient was subjected to lumpectomy. Histopathological examination of the specimen revealed adult microfilaria with characteristic morphology of Wuchereria bancrofti.

\section{DISCUSSION:}

Filarial infection is endemic in the tropics and part of Africa. Breast filariasis has been reported in the Indian subcontinent [1].

Lymphatic filariasis affect an estimated 120 million people worldwide [2].

The disease has equal distribution in both sexes [3]. W bancrofti and B malayi are the predominant cause of lymphatic filariasis, the former being commoner [2].

The clinical manifestations of the disease are due to lymphatic dysfunction, obstruction and inflammation [2].

Usually the diagnosis is made by the detection of microfilaria in the patient's blood. Tests for artificial antibodies or circulating parasite antigen may also be useful [2].

The breast is a recognised site of involvement although infrequent $[4,1]$.

Solitary and superficial palpable nodule in the breast is a common presentation, the upper outer quadrant being a most common site $[2,5,1]$.
On mammograms, elongated, serpentine, non-ductal calcifications without evidence of irregularity or pleomorphism are seen [2,5,3].

Mosquitoes that deposit infective larvae on the skin transmit the infection. The larvae develop into adult worms over a period of six months to two years in the lymphatics.

Adult worm produces microfilaria, which circulate in the blood stream [2].

The adult worms show rigorous movement in the body, which on real time ultrasound shows a characteristic appearance labelled as "filarial dance" $[4,5]$.

This case emphases upon the importance of the classic and diagnostic ultrasound finding of filarial dance, especially in the absence of characteristic serpentine calcification on film mammograms.

\section{REFERENCES:}

1. Rosen P P. Specific infections. In: Rosen P P, ed. Breast Pathology. 2nd ed. Philadelphia: Lippincott Williams and Wilkins, 2001:65-75.

2. Chow C K, McCarthy J, Neafie R.et al. Mammography of lymphatic filariases. AJR1996;167:1425-1427.

3. Friedman P D. Kalisher L.Case 43: Filariases. Radiology 2002;222:515-517.

4. Jungmann P, Figueredo-Silva J. Dreyer G. Bancroftian lymphangitis in northeastern Brazil: a histophathologic study of 17 cases. J Trop Med Hyg 1992;95:114-118.

5. Patil J A, Patil A D, Ramani S K. filarial "Dance" in Breast Mass.AJR 2003;181:1157-1158 